\title{
GABA interneurons mediate the rapid antidepressant- like effects of scopolamine
}

\author{
Eric S. Wohleb, ${ }^{1}$ Min Wu, ${ }^{1}$ Danielle M. Gerhard, ${ }^{2}$ Seth R. Taylor, ${ }^{1}$ Marina R. Picciotto, ${ }^{1}$ Meenakshi Alreja, ${ }^{1}$ and Ronald S. Duman ${ }^{1}$ \\ 'Department of Psychiatry, Yale University School of Medicine, New Haven, Connecticut, USA. ²Department of Psychology, Yale University, New Haven, Connecticut, USA
}

\begin{abstract}
Major depressive disorder (MDD) is a recurring psychiatric illness that causes substantial health and socioeconomic burdens. Clinical reports have revealed that scopolamine, a nonselective muscarinic acetylcholine receptor antagonist, produces rapid antidepressant effects in individuals with MDD. Preclinical models suggest that these rapid antidepressant effects can be recapitulated with blockade of M1-type muscarinic acetylcholine receptors (M1-AChR); however, the cellular mechanisms underlying activity-dependent synaptic and behavioral responses to scopolamine have not been determined. Here, we demonstrate that the antidepressant-like effects of scopolamine are mediated by GABA interneurons in the medial prefrontal cortex (mPFC). Both GABAergic (CAD67+) interneurons and glutamatergic (CaMKII+) interneurons in the mPFC expressed M1-AChR. In mice, viral-mediated knockdown of M1-AChR specifically in CABAergic neurons, but not glutamatergic neurons, in the $\mathrm{MPFC}$ attenuated the antidepressant-like effects of scopolamine. Immunohistology and electrophysiology showed that somatostatin (SST) interneurons in the mPFC express M1-AChR at higher levels than parvalbumin interneurons. Moreover, knockdown of M1-AChR in SST interneurons in the mPFC demonstrated that M1-AChR expression in these neurons is required for the rapid antidepressant-like effects of scopolamine. These data indicate that SST interneurons in the $\mathrm{mPFC}$ are a promising pharmacological target for developing rapid-acting antidepressant therapies.
\end{abstract}

\section{Introduction}

Major depressive disorder (MDD) is a recurring neuropsychiatric illness that affects up to $17 \%$ of the population and causes substantial social and economic burdens (1-4). While some patients respond to existing medications, currently available antidepressants take weeks to months to have an effect, and many patients are considered treatment resistant because they fail to respond to 2 or more antidepressants (5). Recent studies demonstrate that scopolamine, a nonselective muscarinic acetylcholine (ACh) receptor (mAChR) antagonist, and ketamine, an NMDA receptor antagonist, produce rapid antidepressant actions (within hours) and are effective even in treatment-resistant MDD patients (6-8). The rapid antidepressant actions of scopolamine and ketamine are dependent on glutamate release and induction of new spine synapses in the medial prefrontal cortex (MPFC) (9-11), effects that directly target the synaptic pathophysiology of MDD and chronic stress (12-19).

However, a major question in the field is, what are the initial cellular targets that mediate the increase in glutamate transmission, leading to increased synapse formation and rapid antidepressant behavior $(10,20,21)$ ? One hypothesis is that scopolamine blocks muscarinic receptors on GABAergic interneurons, resulting in disinhibition of pyramidal neurons and increased glutamate transmission. Alternatively, scopolamine may act directly on pyramidal neurons to enhance synaptic plasticity and produce antidepressant behavioral responses. Recent studies suggest that the M1-type muscarinic ACh receptor (M1-AChR) subtype may mediate the antidepressant actions of scopolamine $(9,22,23)$.

Conflict of interest: The authors have declared that no conflict of interest exists Submitted: October 8, 2015; Accepted: April 14, 2016.

Reference information: J Clin Invest. 2016;126(7):2482-2494. doi:10.1172/JCI85033.
M1-AChRs are expressed on both GABAergic interneurons and glutamatergic pyramidal neurons in the $\mathrm{MPFC}$ and regulate the activity of both cell types (24-26).

In the present study, we used transgenic mice and viralmediated gene transfer to drive Cre-dependent expression of M1-AChR shRNA in different subtypes of GABA interneurons or glutamate neurons in the $\mathrm{MPFC}$. The results demonstrate that M1-AChR knockdown in GABA interneurons, but not pyramidal neurons, blocks the antidepressant-like response to scopolamine in mouse behavioral models. In addition, we show that knockdown of M1-AChR in somatostatin (SST), but not parvalbumin (PV), interneurons, blocks the actions of scopolamine. These findings reveal that M1-AChRs on SST interneurons in the MPFC are a critical cellular target underlying the rapid antidepressant effects of scopolamine.

\section{Results}

CaMKII ${ }^{+}$and $\mathrm{GAD}^{+}$cell type-specific knockdown of M1-AChR in the $m P F C$. To determine whether glutamatergic pyramidal neurons and GABAergic interneurons in the $\mathrm{MPFC}$ express M1-AChR, double immunohistology was conducted with an M1-AChR antibody and $\mathrm{Ca}^{2+} /$ calmodulin-dependent kinase II (CaMKII; pyramidal cell) or glutamate decarboxylase-67 (GAD67; GABA interneuron) (25). The results show that $\mathrm{CaMKII}^{+}$pyramidal neurons display punctate M1-AChR labeling in the MPFC, and higher magnification shows the presence of M1-AChR ${ }^{+}$neurons that lack CaMKII labeling (Figure 1, A and B). Double labeling with GAD67 shows that many of the $\mathrm{CaMKII}^{-}$neurons were GABA interneurons (Figure $1 \mathrm{C}$ ).

For cell-specific M1-AChR knockdown, we used a construct that expresses shRNA targeting M1-AChR (M1shRNA) in a Crerecombinase-dependent manner (Supplemental Figure 1A). Both 
A

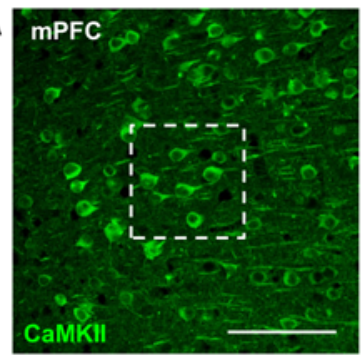

B

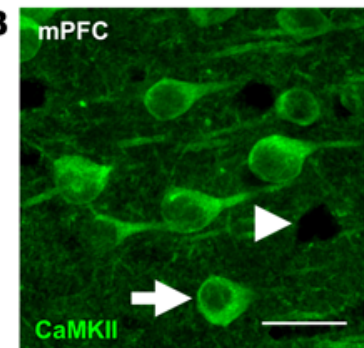

C

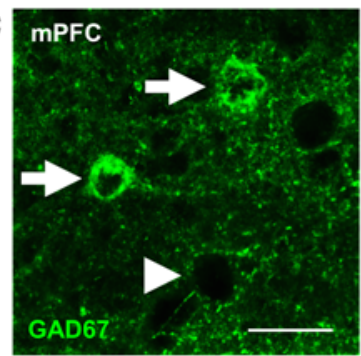

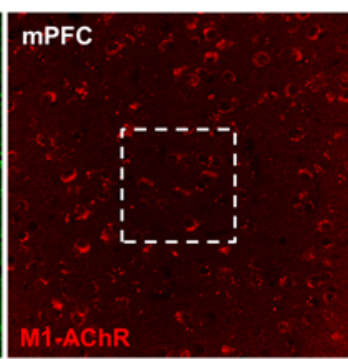
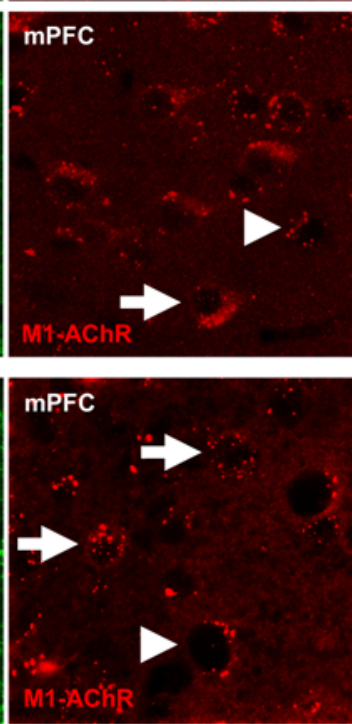
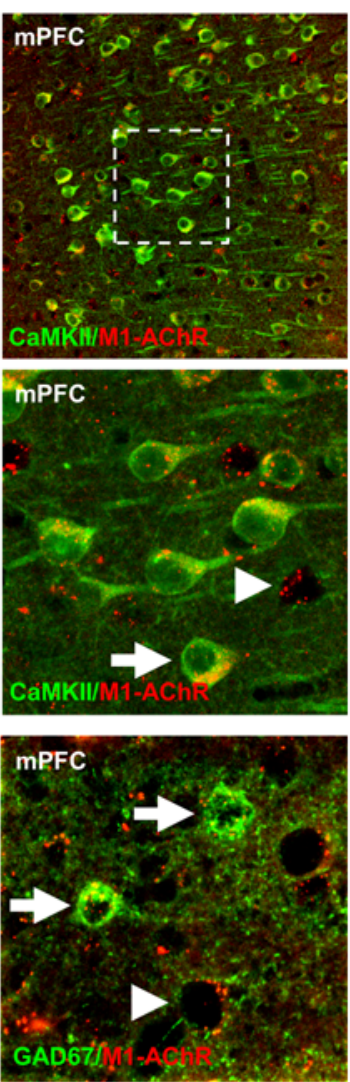

Figure 1. M1-AChR colocalized with CaMKII+ and GAD67 neurons in the MPFC. WT mice were perfused, and brains were processed for immunohistology. Representative confocal images of immunofluorescent labeling in the MPFC are shown. (A) Images of CaMKII (green) and M1-AChR (red) in the MPFC are shown. Original magnification, $\times 40$. Scale bar: $100 \mu \mathrm{m}$. (B) Magnified images of the dashed portion are shown. Original magnification, $x 40$, zoom 3. Scale bar: $25 \mu \mathrm{m}$. (C) Subsequent sections labeled with GAD67 (green) and M1-AChR (red) are shown. Original magnification, $\times 40$, zoom 3. Scale bar: $25 \mu \mathrm{m}$. Arrows show neurons colabeled for specific marker and M1-AChR, while arrowheads show neurons that only label with M1-AChR.
DsRed and EGFP are expressed unless recombination deletes EGFP and allows expression of only DsRed and M1shRNA. To test this construct, N2a cells were cotransfected with Cre-expressing (CAG-Cre) and M1shRNA plasmids (pM1shRNA). Transfection with pM1shRNA alone resulted in EGFP and DsRed expression (Supplemental Figure 1B), while cotransfection with CAG-Cre and pM1shRNA resulted in recombination, shown by reduced EGFP expression and retained DsRed expression (Supplemental Figure 1C). Cotransfection with CAG-Cre decreased EGFP mRNA levels in control and pM1shRNA cultures (main effect, $\mathrm{F}_{1,14}=38.86$, $P<0.0001$, Supplemental Figure 1D) and, importantly, resulted in robust knockdown of M1-AChR mRNA (interaction, $\mathrm{F}_{1,22}=16.07$, $P<0.0006$, Supplemental Figure 1E). There was no significant effect on mAChR subtypes (mAChR2-5), demonstrating specificity of the M1shRNA (Supplemental Figure 1F).

Knockdown of M1-AChR in $\mathrm{GAD}^{+}$, but not CaMKII+, neurons blocks the antidepressant effects of scopolamine. pM1shRNA was packaged into an adeno-associated virus-2 (AAV2 ${ }^{\mathrm{M} 1 \mathrm{shRNA}}$ ) and infused bilaterally into the mPFC of Gad1-Cre and Camk2a-Cre mice or their WT littermate controls. The MPFC was targeted, as prior studies show direct infusion of scopolamine into this region caused antidepressant actions. Initial studies were conducted in Gad1-Cre mice, and 3 weeks after viral infusion, brains were collected for histology (Figure 2A). Figure 2B shows the spread of AAV2 ${ }^{\mathrm{M} 1 s h R N A}$ infusion in the $\mathrm{MPFC}$, and higher magnification shows that there was no observed recombination in WT mice (Figure 2C). However, infusion with AAV2 ${ }^{\mathrm{M} 1 \text { shRNA }}$ into Gad1-Cre mice resulted in a subset of cells displaying DsRed only (Figure 2C), demonstrating cell-specific expression of the M1shRNA construct.
Behavioral studies using the same design demonstrate that infusions of AAV2 ${ }^{\mathrm{M} 1 \mathrm{shRNA}}$ into Gad1-Cre mice had no significant effects on baseline behaviors in the open field test (total distance and center time, anxiety measure) or forced swim test (FST) (immobility, behavioral despair measure) compared with those of WT controls receiving the same viral infusions (Figure 2, $\mathrm{E}-\mathrm{G})$. Mice then received 3 doses of scopolamine $(25 \mu \mathrm{g} / \mathrm{kg}$, i.p. $)$ every other day, a dosing regimen based on the schedule used in depressed human subjects and shown to produce robust signaling and behavioral responses in rodents (23). In the FST, scopolamine significantly decreased immobility time in WT mice infused with AAV2 ${ }^{\text {M1shRNA }}$, but these effects were completely blocked in Gad1Cre mice receiving AAV2 ${ }^{\mathrm{M} 1 s h R N A}$ infusions into the $\mathrm{MPFC}$ (interaction, $F_{1,28}=8.17, P<0.0008$; Figure $2 \mathrm{H}$ ). In the novelty suppressed feeding test (NSFT), scopolamine decreased latency to feed in WT mice infused with AAV2 ${ }^{\mathrm{M} 1 \text { shRNA }}$, but again had no effect in Gad1Cre mice receiving the same viral infusions (main effect of scopolamine, $\mathrm{F}_{1,28}=6.44, P<0.02$; main effect of genotype, $\mathrm{F}_{1,28}=3.88$, $P<0.06$; interaction, $\mathrm{F}_{1,28}=2.71, P<0.11$; Figure 2I).

Similar studies were conducted in Camk2a-Cre mice with infusions of AAV2 ${ }^{\mathrm{M} 1 \text { shRNA }}$ in the $\mathrm{MPFC}$ (Figure 3, A and B). There was no recombination observed in WT mice infused with AAV2 ${ }^{\text {M1shRNA }}$ (Figure 3, B and C), but in Camk2a-Cre mice, the majority of neurons showed recombination (DsRed ${ }^{+}$only) due to the high density of glutamatergic pyramidal neurons in mPFC (Figure 3D). Behavioral testing showed that AAV2 ${ }^{\mathrm{M} 1 \text { shRNA }}$ had no effect on baseline behavior in the open field test (distance or center time) or immobility in the FST (Figure 3, E-G). Scopolamine administration (3-dose regimen) significantly decreased immobility in the FST in both WT 
A

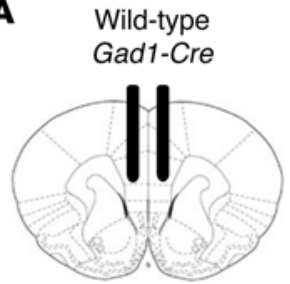

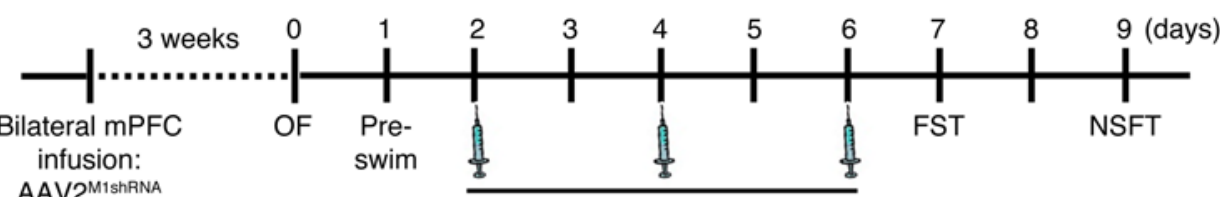

Saline or scopolamine $(25 \mu \mathrm{gkg})$

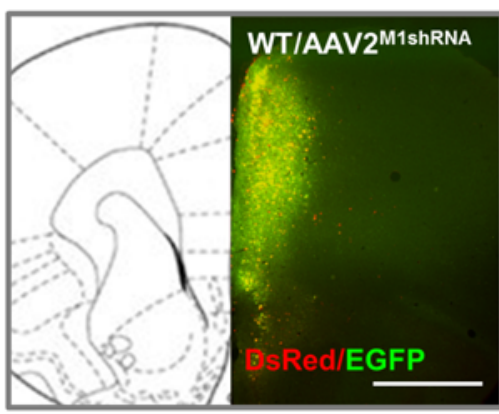

c

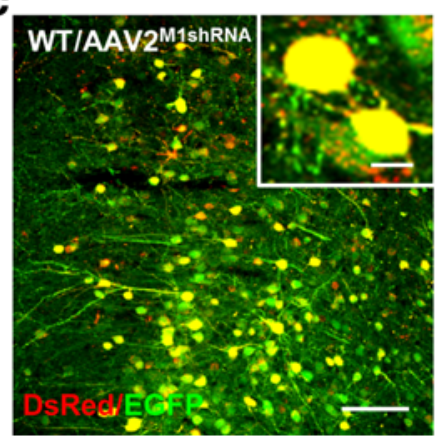

D

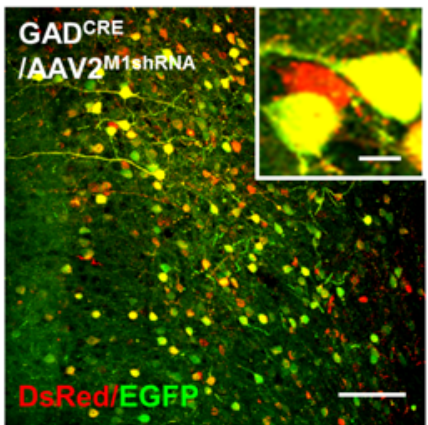

E

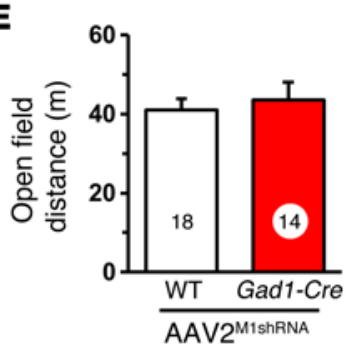

$\mathbf{F}$

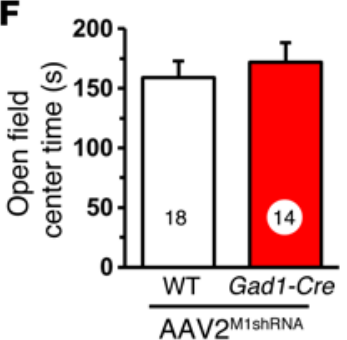

G

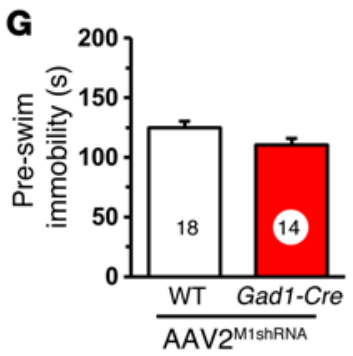

H

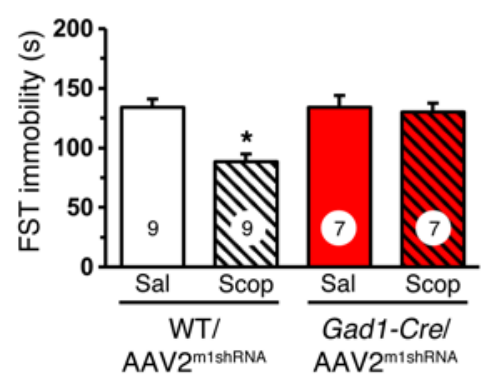

I

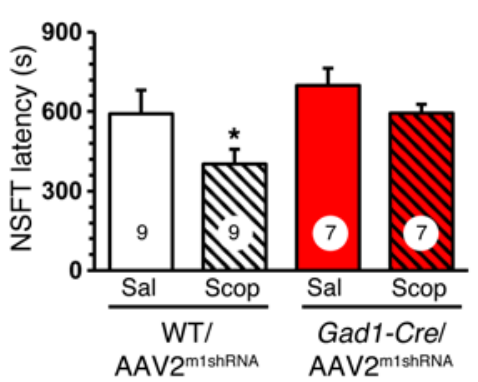

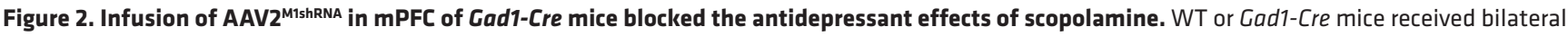

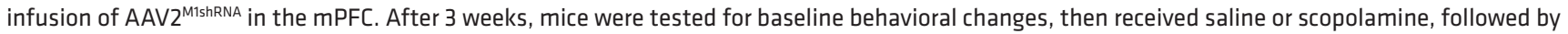
additional behavioral tests. (A) Schematic showing experimental approach and time line. A subset of mice were perfused, and brains were processed for histology. Representative confocal images of fluorescence in the mPFC are shown. OF, open field. (B) Low magnification image in WT/AAV2 ${ }^{\text {MrhhRNA }}$ mouse to show representative infusion in mPFC. Original magnification, $\times 4$. Scale bar: $1 \mathrm{~mm}$. (C) Representative image of AAV2 ${ }^{\mathrm{MishRNA}}$ in prelimbic mPFC of WT mouse. Original magnification, $\times 20$. Inset shows all neurons have colocalization of eGFP (green) and DsRed (red) fluorescence. Scale bars: $100 \mu \mathrm{m} ; 10 \mu \mathrm{m}$

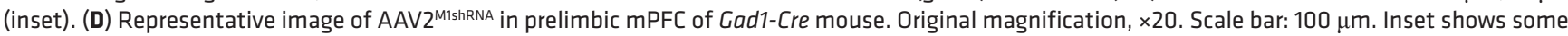
neurons have colocalization of eGFP (green) and DsRed (red) fluorescence, while others only have DsRed fluorescence. Scale bar: $10 \mu \mathrm{m}$. Prior to scopolamine treatment, mice were tested in open-field activity and FST (preswim). Total distance in the open field (E), time spent in center of open field (F), and time spent immobile in the preswim are shown (C) for each set of experiments. Following repeated doses of scopolamine, WT or Gad1-Cre mice were tested in FST and NSFT. Time spent immobile in the FST (H) and latency to feed in NSFT are shown (I). Scop, scopolamine; Sal, saline. Bars represent the mean \pm SEM, $n=7-9$ /group. Numbers in the bars represent the total sample size for each group. ${ }^{*} P<0.05$, means significantly different from the respective saline group based on ANOVA.

and Camk2a-Cre mice infused with AAV2 $2^{\mathrm{M} \text { shRNA }}$ (main effect, $\mathrm{F}_{1,32}=$ $18.58, P<0.0001$, Figure $3 \mathrm{H}$ ). Similar results were observed in the NSFT, where scopolamine administration reduced the latency to feed in both WT and Camk2a-Cre mice receiving AAV2 ${ }^{\mathrm{MishRNA}}$ infusion (main effect, $\mathrm{F}_{1,35}=27.28, P<0.0001$, Figure 3I).
M1-AChR knockdown in GAD interneurons blocks scopolamine stimulation of FosB. Prior studies indicate that scopolamine increases Fos labeling in the MPFC, further evidence of increased glutamate neurotransmission and neuronal activation (23). To determine whether M1-AChR knockdown blocks scopolamine 
A

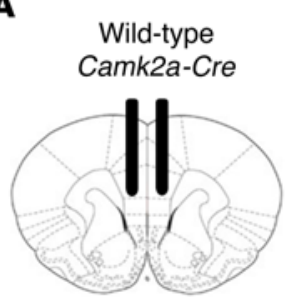

B

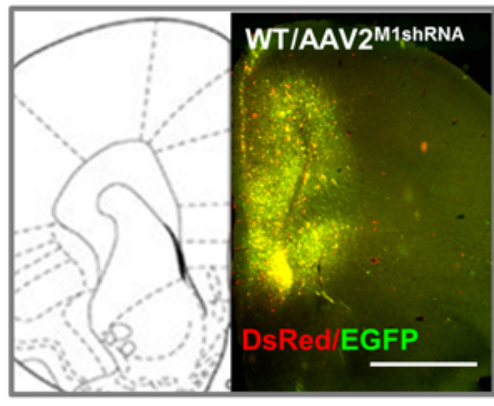

C

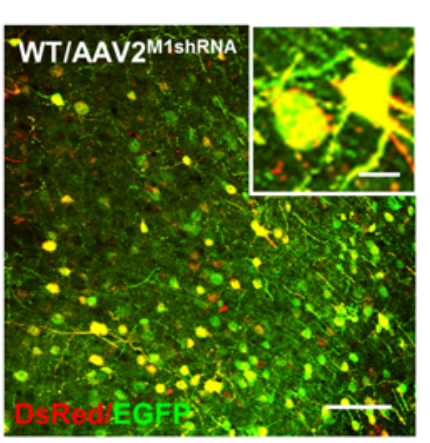

D

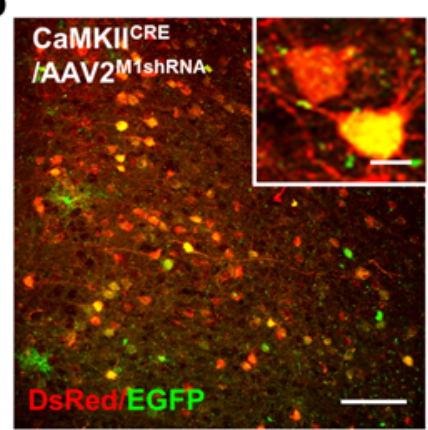

E

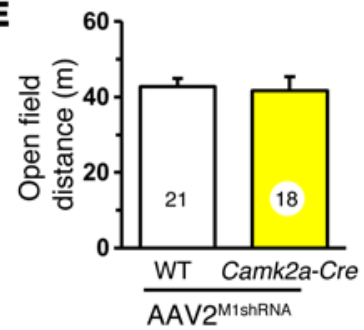

$\mathbf{F}$

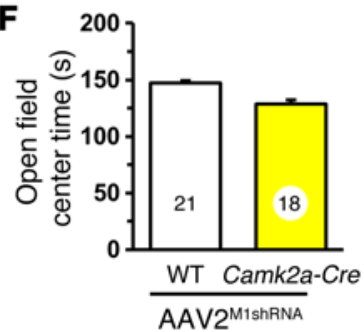

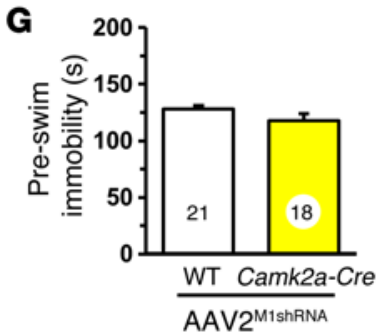

H

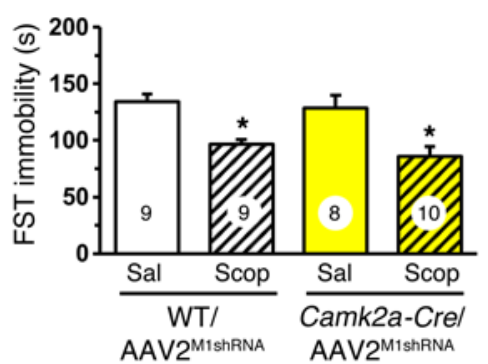

I

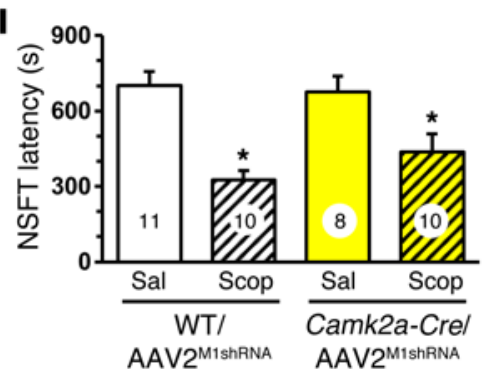

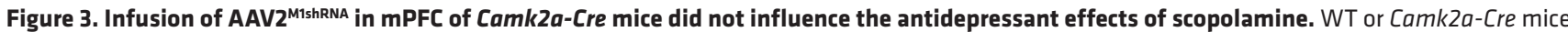
received bilateral infusion of $A A V 2^{\mathrm{M} 1 s h R N A}$ in the $\mathrm{mPFC}$. After 3 weeks, mice were tested for baseline behavioral changes, then received saline or scopolamine, followed by additional behavioral tests. (A) Schematic showing experimental approach and time line. A subset of mice were perfused, and brains were processed for histology. Representative confocal images of fluorescence in the mPFC are shown. (B) Low-magnification image in WT/AAV2 ${ }^{\text {MishRNA }}$ mouse to show representative infusion in mPFC. Original magnification, $\times 4$. Scale bar: $1 \mathrm{~mm}$. (C) Representative image of AAV2 ${ }^{\mathrm{MishRNA}}$ in prelimbic mPFC of WT mouse. Original magnification, $\times 20$. Inset shows all neurons have colocalization of EGFP (green) and DsRed (red) fluorescence. Scale bars: $100 \mu \mathrm{m}$; $10 \mu \mathrm{m}$ (inset). (D) Representative image of $A A V 2^{\mathrm{M} / s h R N A}$ in prelimbic mPFC of Camk2a-Cre mouse. Original magnification, $\times 20$. Inset shows that many neurons have only DsRed fluorescence, while few have colocalization of EGFP (green) and DsRed (red) fluorescence. Scale bars: $100 \mu \mathrm{m} ; 10 \mu \mathrm{m}$ (inset). Prior to scopolamine treatment, mice were tested in open-field activity and FST (preswim). Total distance in the open field (E), time spent in center of open field $(\mathbf{F})$, and time spent immobile in the preswim are shown (G) for each set of experiments. Following repeated doses of scopolamine, WT or Gad1-Cre mice were tested in FST and NSFT. Time spent immobile in the FST (H) and latency to feed in NSFT are shown (I). Bars represent mean \pm SEM, $n=8-11 /$ group. ${ }^{*} P<0.05$, means significantly different from respective saline group based on ANOVA. Numbers in the bars represent the total sample size for each group.

induction of neuronal activation in the $\mathrm{mPFC}$, we examined FosB immunohistology (27). WT and Gad1-Cre mice infused with AAV2 ${ }^{\text {MishRNA }}$ received a final injection of scopolamine $(25 \mu \mathrm{g} / \mathrm{kg})$, and then 1 hour later, brains were collected and processed for FosB immunohistology. Scopolamine increased the number of $\mathrm{FosB}^{+}$ neurons in the mPFC of WT, but not Gad1-Cre, mice following $\mathrm{AAV}^{\mathrm{M} 1 \text { shRRNA }}$ infusions (Figure 4A). Scopolamine induction of FosB ${ }^{+}$ neurons was observed in both the infralimbic and prelimbic cortices of WT/AAV2 ${ }^{\text {MishRNA }}$, but not in Gad1-Cre/AAV2 ${ }^{\text {MishRNA }}$ mice (infralimbic, interaction, $\mathrm{F}_{1,14}=17.51, P<0.0009$; prelimbic, interaction, $\mathrm{F}_{1,14}=4.22, P<0.06$; Figure 4, B and C). Studies of Camk2aCre mice showed that scopolamine induction of $\mathrm{FosB}^{+}$neurons was observed in both WT/AAV2 ${ }^{\mathrm{M} 1 h \mathrm{RNA}}$ and Camk2a-Cre/AAV2 ${ }^{\mathrm{MishRNA}}$ mice (infralimbic, main effect, $\mathrm{F}_{1,12}=83.09, P<0.0001$; prelim- 


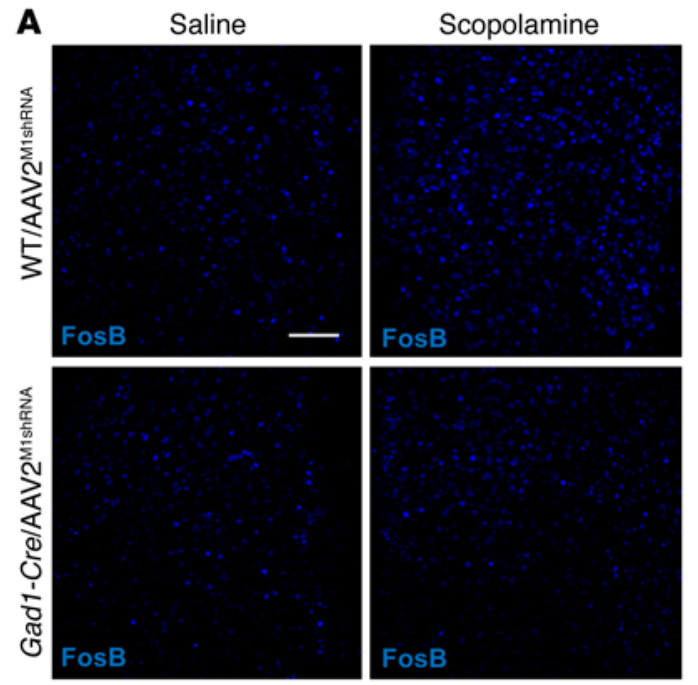

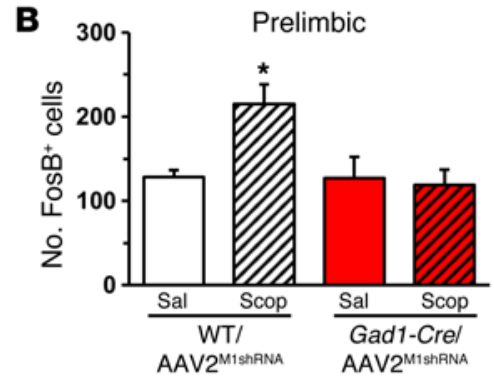

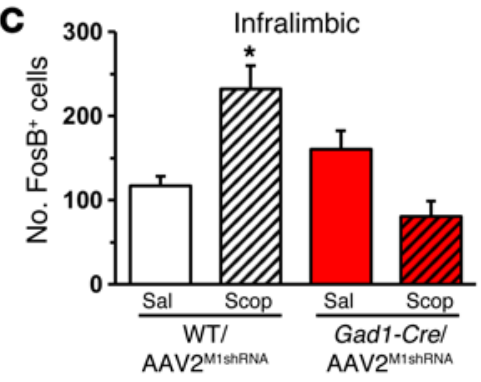

Figure 4. M1-AChR knockdown in Gad1-Cre mice decreased FosB activation following scopolamine treatment. WT or Gad1-Cre mice received bilateral infusion of $A A V 2^{\text {MishRNA }}$ in the $\mathrm{mPFC}$. Following behavioral tests, mice received an acute scopolamine injection $(25 \mu \mathrm{g} / \mathrm{kg})$ and were perfused 1 hour later. Brains were collected and processed for immunohistology. (A) Representative images of FosB labeling in the prelimbic mPFC of WT/AAV2 ${ }^{\text {MishRNA }}$ and Gad1-Cre/AAV2 ${ }^{\text {MishRNA }}$ mice treated with saline or scopolamine. Scale bar: $100 \mu \mathrm{m}$. (B) Quantification of FosB ${ }^{+}$neurons in the (B) prelimbic or (C) infralimbic mPFC. Bars represent the mean \pm SEM, $n=4-5 /$ group. ${ }^{*} P<0.05$, means significantly different from respective saline group based on ANOVA. bic, main effect, $\mathrm{F}_{1,12}=67.52, P<0.0001$; Supplemental Figure 3). Knockdown of M1-AChR was confirmed by immunolabeling studies that showed that Camk2a-Cre/AAV2 ${ }^{\mathrm{M} 1 s h \mathrm{RNA}}$ significantly reduced M1-AChR immunolabeling in pyramidal neurons compared with WT/AAV2 ${ }^{\text {MishRNA }}$ mice $(P<0.02$, Supplemental Figure $2)$. These findings provide further evidence for functional knockdown of M1-AChR in Gad1-Cre mice and blockade of scopolamineinduced neuronal activity.

$P V$ and SST interneurons show varied distribution and expression of M1-AChR in the MPFC. GABAergic interneurons in the PFC have diverse physiological properties and can be categorized into subtypes based on expression of calcium-binding proteins, such as PV, and neuropeptides, such as SST. Each interneuron subtype has a dynamic role in modulating the activity of glutamatergic pyramidal cells based on firing rates and synaptic connections $(28,29)$. To further characterize the role of GABA interneurons in the $\mathrm{mPFC}$, we used double immunohistology to determine distribution and colocalize expression of M1-AChR on PV and SST interneuron subtypes (Figure 5, A and B). PV interneurons were concentrated in deeper cortical layers (III-V), while SST interneurons were dispersed evenly across cortical layers (Supplemental Figure 4 and Figure 5D). Further analyses showed that M1-AChR is more frequently colocalized with SST compared with PV interneurons, with approximately $62 \%$ of SST and $24 \%$ of PV interneurons colocalized with M1-AChR across both $\mathrm{MPFC}$ regions (Figure 5, C and D).

M1-AChR mediates cholinergic stimulation of SST interneurons in the $m P F C$. To determine the role of M1-AChR in SST interneuron responses to cholinergic stimulation, brain slice electrophysiology was performed with SST-tdTomato mice. ACh or muscarine was applied to MPFC SST interneurons in $\mathrm{MPFC}$ slices under wholecell voltage clamp (layer II/III and V). The SST-tdTomato interneurons had a mean resting membrane potential equal to $-75.4 \pm 1.4$ $\mathrm{mV}$, and ACh induced an inward current (mean $=85.7 \pm 17.8 \mathrm{pA}$ ). Muscarine application also produced an inward current (mean = $43.3 \pm 9.9 \mathrm{pA}$ ), while nicotine application caused no change in SSTtdTomato interneuron currents (Figure 6A). In all, 92\% of SST-
tdTomato interneurons showed inward current responses. We also found that SST-tdTomato interneurons displayed increased excitatory postsynaptic currents (EPSCs) after muscarine application, as a result of excitatory inputs from pyramidal neurons (Figure 6B). Prior application of the $\mathrm{mACh}$ receptor antagonist telenzepine blocked both muscarine-induced inward current (musc: mean = $40 \pm 9.6 \mathrm{pA}$; telenzepine + musc: mean $=0 \pm 0 \mathrm{pA}$, Figure $6 \mathrm{~B}$ ) and EPSCs in SST-tdTomato interneurons (Figure 6C).

To confirm neuron subtype-specific knockdown of M1-AChR, SST interneurons from Sst-Cre/AAV2 ${ }^{\mathrm{M} 1 \text { shRNA }}$ mice were recorded (Figure 6D). DsRed ${ }^{+}$SST interneurons in these mice had resting membrane potentials equal to $-77.3 \pm 2.8 \mathrm{mV}$, comparable to SST-tdTomato interneurons shown above. In Sst-Cre/AAV2 ${ }^{\text {M1shRNA }}$ mice, a minimal muscarine-induced inward current was observed in 1 out of 6 (17\%) interneurons tested. Muscarine-induced EPSCs were observed in 5 out of 6 (83\%) interneurons tested (Figure $6 \mathrm{E}$ ), indicating that synaptic inputs onto SST interneurons were not affected in Sst-Cre/AAV2 ${ }^{\mathrm{M} 1 \text { shRNA }}$ mice. These EPSCs were blocked when telenzepine was applied prior to muscarine (Figure $6 \mathrm{E})$. Collectively, these data show that cholinergic stimulation of SST interneurons in the MPFC is mediated by M1-AChR and that AAV2 ${ }^{\text {M1shRNA }}$ effectively blocked muscarinic stimulation of SST interneurons. Knockdown of M1-AChR was confirmed by immunolabeling studies, which showed that infusion of AAV2 ${ }^{\text {M1shRNA }}$ into Sst-Cre mice significantly reduced M1-AChR immunolabeling compared with that of Sst-Cre mice infused with a scrambled shRNA control (AAV2 $\left.{ }^{\mathrm{SCR}}\right)(P<0.02$, Supplemental Figure 2$)$.

Knockdown of M1-AChR in SST, but not PV, interneurons prevents the antidepressant-like effects of scopolamine. To determine the GABAergic interneuron subtype that mediates the behavioral responses to scopolamine, WT, Sst-Cre, and PV-Cre mice received bilateral infusion of $A A V 2^{\mathrm{M} 1 \text { shRNA }}$ into the $\mathrm{MPFC}$ and were analyzed 3 weeks later (Figure 7A). Infusion of AAV2 ${ }^{\mathrm{M} 1 \text { shRNA }}$ into the $\mathrm{MPFC}$ of Sst-Cre mice resulted in recombination in a subset of neurons, shown by expression of only DsRed (Figure 7, B and C). AAV2 ${ }^{\mathrm{M} 1 \text { shRNA }}$ infusion in Sst-Cre mice produced no significant 
A
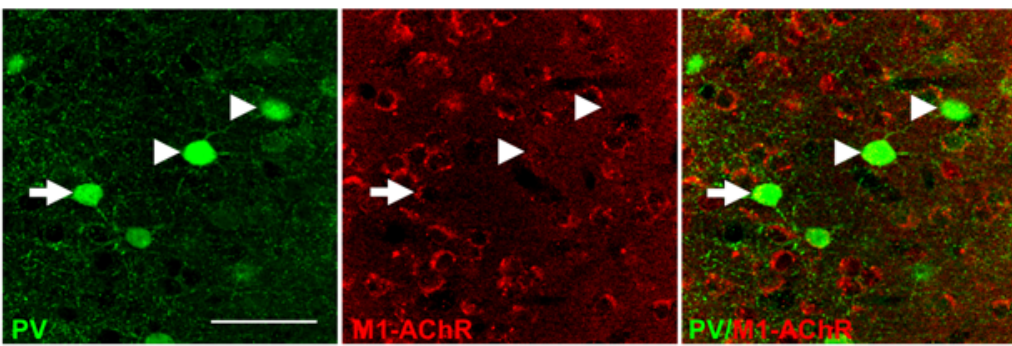

B
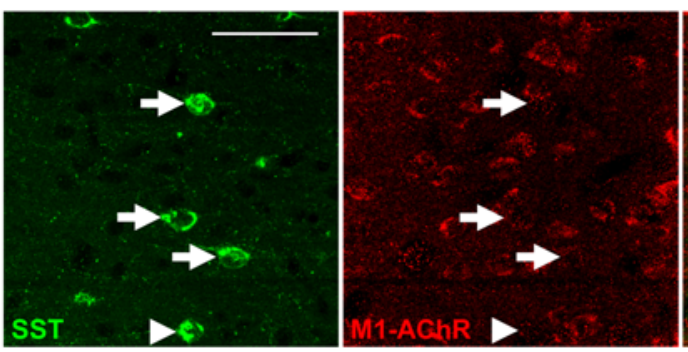

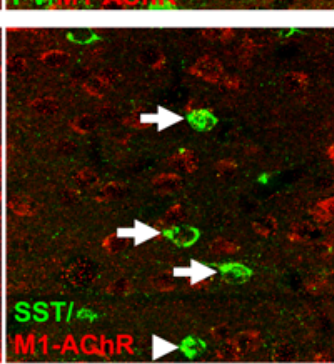

C

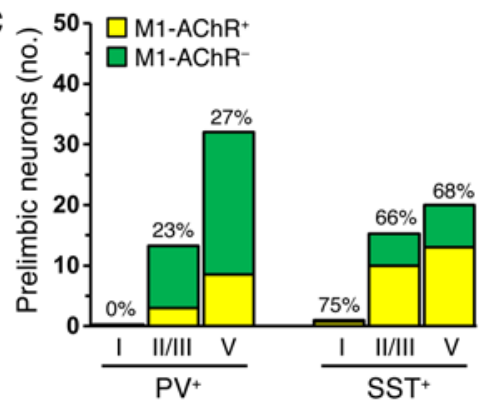

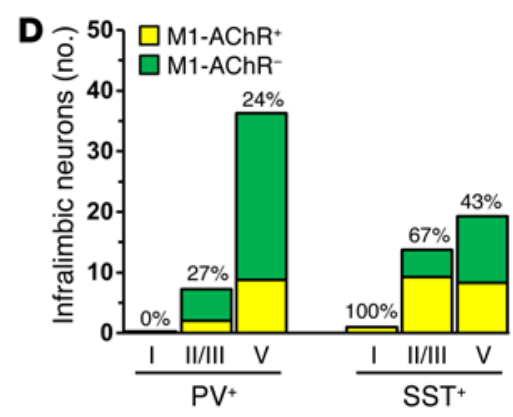

Figure 5. Parvalbumin and SST interneurons have varied expression of M1-AChR in the MPFC. WT mice were perfused, and brains were processed for immunohistology. Representative confocal images of immunofluorescent labeling in the MPFC are shown. (A) Images of PV (green) and M1-AChR (red) in the MPFC are shown. Original magnification, $\times 40$, zoom 2. (B) Images of SST (green) and M1-AChR (red) in the MPFC are shown. Original magnification, $\times 40$, zoom 2. Scale bars: $50 \mu \mathrm{m}$. Arrows show interneurons colabeled for specific marker and M1-AChR, while arrowheads show interneurons that do not colabel with M1-AChR. (C and D) Number and proportion of PV and SST interneurons that colocalized with M1-AChR in layers I, II/III, and V of the (C) prelimbic or (D) infralimbic mPFC. Proportions represent the percentage of M1-AChR ${ }^{+}$interneurons in each subpopulation and cortical layer indicated. changes in baseline behavior other than a small increase in preswim immobility compared with that of WT mice $(P<0.003$; Supplemental Figure 5, B-D). In littermate WT/AAV2 ${ }^{\mathrm{M} 1 \text { shRNA }}$ mice, scopolamine reduced immobility in the FST as expected, but this effect was completely absent in Sst-Cre/AAV2 ${ }^{\mathrm{M} 1 s h \mathrm{RNA}}$ mice (interaction, $\mathrm{F}_{1,27}=7.95, P<0.009$; Figure 7E). In the NSFT, scopolamine decreased latency to feed in WT/AAV2 ${ }^{\mathrm{M} 1 \text { shRNA }}$ mice, but had no effect in Sst-Cre $/ \mathrm{AAV} 2^{\mathrm{M} 1 \text { shRNA }}$ mice (interaction, $\mathrm{F}_{1,26}=3.75$, $P=0.06$, Figure $7 \mathrm{~F})$. In $\mathrm{PV}$-Cre mice, infusion of AAV2 ${ }^{\mathrm{M} 1 \text { shRNA }}$ resulted in recombination in a subset of neurons, shown by expression of DsRed only (Figure 7D). M1-AChR knockdown in PV-Cre mice caused no baseline behavioral effects in either the open field test or FST (Supplemental Figure 5, E-G) and had no effect on the antidepressant-like actions of scopolamine in the FST (main effect, $\mathrm{F}_{1,23}=22.35, P<0.0001$, Figure $7 \mathrm{G}$ ) or NSFT (main effect, $\mathrm{F}_{1,24}=13.12, P<0.001$, Figure $7 \mathrm{H}$ ). Following behavioral testing, studies were conducted to determine whether M1-AChR knockdown in SST interneurons blocks scopolamine induction of FosB. In WT/AAV2 ${ }^{\mathrm{M} 1 \text { shRNA }}$ mice, scopolamine increased $\mathrm{FosB}^{+}$staining in the $\mathrm{mPFC}$, but there were no significant differences in Sst-Cre/ AAV2 ${ }^{\mathrm{M} 1 \text { shRNA }}$ mice (Figure 8A). Scopolamine increased the number of $\mathrm{FosB}^{+}$neurons in both the prelimbic (interaction, $\mathrm{F}_{1,14}=21.64, P<$ 0.004 ; Figure $8 \mathrm{~B}$ ) and infralimbic (interaction, $\mathrm{F}_{1,14}=7.68, P<0.01$; Figure $8 \mathrm{C}$ ) cortices of WT/AAV2 ${ }^{\mathrm{M} 1 \text { shRNA }}$ mice, but there were no significant effects in Sst-Cre/AAV2 ${ }^{\mathrm{M} 1 s h R N A}$ mice (Figure 8, B and C).

To test the possibility that the effects of AAV2 ${ }^{\text {M1shRNA }}$ knockdown might occur via nonspecific actions of shRNA expression, we examined the effects of a scrambled shRNA control (AAV2 ${ }^{\mathrm{SCR}}$ ) in the mPFC of Sst-Cre mice. Immunohistology showed that M1-AChR knockdown occurred in Sst-Cre/AAV2 ${ }^{\text {MishRNA }}$ mice $(P<0.003$, Supplemental Figure 2). Similarly to what occurred in prior studies (Figure 7, E and F), Sst-Cre/AAV2 ${ }^{\mathrm{M} 1 \text { shRNA }}$ mice showed no antidepressant-like response in the FST following scopolamine treatment (Figure 9, A-C). In contrast, Sst-Cre/AAV2 ${ }^{\mathrm{SCR}}$ mice showed antidepressant-like responses comparable to those of WT/AAV2 ${ }^{\text {M1shRNA }}$ mice, with significant reductions in FST immobility (interaction, $\mathrm{F}_{2,27}=5.15, P<0.01$, Figure 9B). In the NSFT, no significant interaction was observed; however, there was a trend for an effect of scopolamine across groups (main effect, $\mathrm{F}_{1,27}=3.29$, $P=0.08$, Supplemental Figure 6). Ad hoc analyses showed that WT/AAV2 ${ }^{\mathrm{M} 1 \text { shRNA }}$ and Sst-Cre/AAV2 ${ }^{\mathrm{SCR}}$ mice treated with scopolamine exhibited a decrease in latency to feed $(P<0.02$ for both, Supplemental Figure 6).

We have also conducted an additional test that examines the antidepressant-like effects of scopolamine in a rodent model of anhedonia, a core symptom of depression. The female urine-sniffing test (FUST) is based on the attraction of male rodents to pleasurable pheromones in urine and serves as a measure of rewardseeking behavior (30). For FUST, the mice are first exposed to a cotton tip dipped in water and then exposed to another cotton tip with fresh urine collected from same-strain females. The results demonstrate that scopolamine administration to WT/AAV2 ${ }^{\text {M1shRNA }}$ and Sst-Cre/AAV2 ${ }^{\mathrm{SCR}}$ mice results in a significant increase in interaction time spent with the female urine (interaction, $\mathrm{F}_{2,26}=3.92, P$ $<0.03$, Figure 9C); there was no effect on time spent with the water (data not shown). In contrast, Sst-Cre/AAV2 ${ }^{\mathrm{M} 1 \text { shRNA }}$ mice adminis- 
A SST-tdTomato

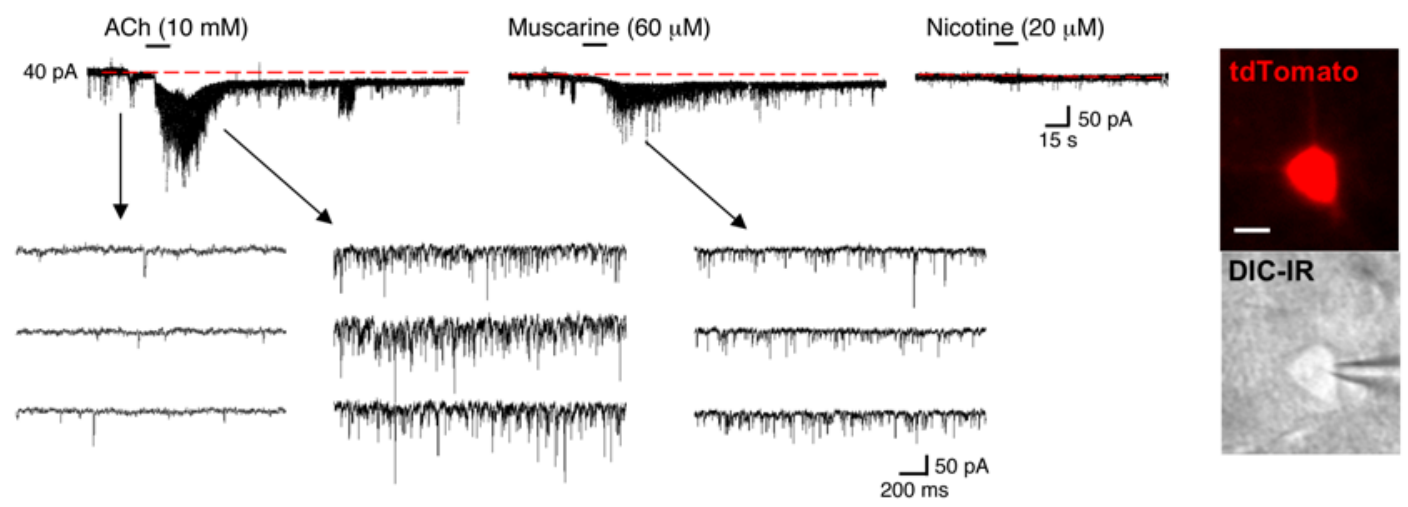

B SST-tdTomato
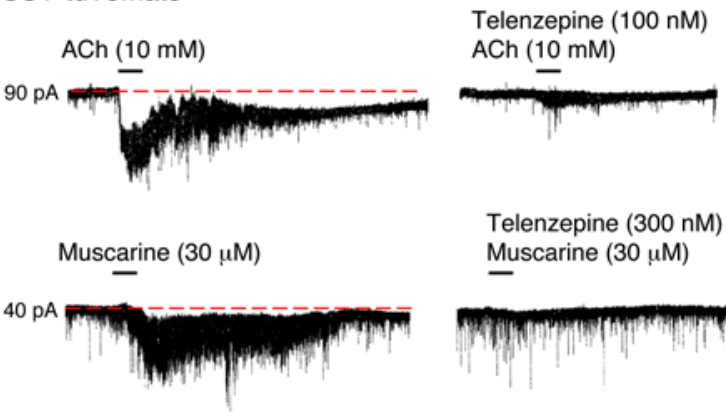

Telenzepine (300 nM)

Muscarine $(30 \mu \mathrm{M})$

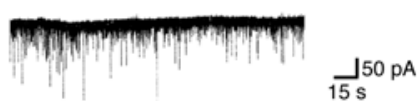

C SST-tdTomato

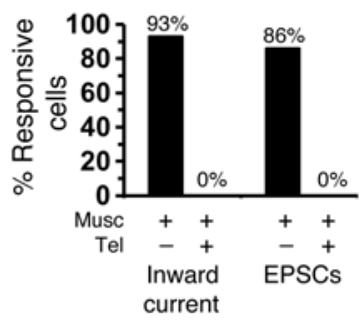

Neurophysiology was performed on DsRed SST interneurons

$$
\text { Bilateral }
$$
mPFC infusion: AAV2 ${ }^{\text {M1shANA }}$

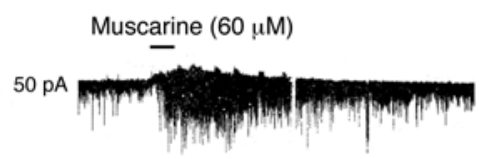

E Sst-Cre/AAV2 $2^{\text {mishRNA }}$

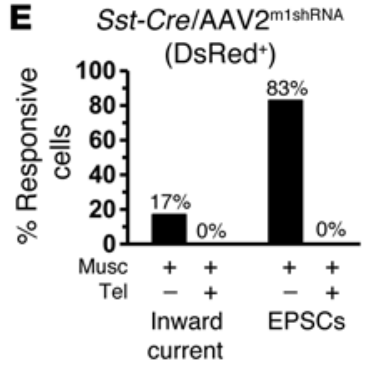

Figure 6. M1-AChR mediates cholinergic stimulation of SST interneurons in the mPFC. SST-tdTomato and Sst-Cre/AAV2 ${ }^{\text {MshRNA }}$ mice were used for brain slice electrophysiology. (A) Representative electrophysiology traces from SST-tdTomato interneurons in the mPFC following application of ACh, muscarine, or nicotine. Consecutive traces shown in the lower panel depict the spontaneously occurring excitatory, inward synaptic currents recorded at $-70 \mathrm{mV}$ in the absence and presence of agonists. Representative image of SST-tdTomato neuron with whole cell patch clamp. Scale bar: $10 \mu \mathrm{m}$. (B) Representative electrophysiology traces from SST-tdTomato interneurons in the mPFC after application of ACh alone or telenzepine followed by ACh. Lower traces show SST-tdTomato interneurons in the mPFC after application of muscarine alone or telenzepine followed by muscarine. (C) Proportion of SST-tdTomato interneurons that exhibited inward current and EPSCs following stimulation with muscarine $(n=14)$ or telenzepine followed by muscarine $(n=6)$. (D) Schematic and representative electrophysiology traces from DsRed interneurons in the mPFC of Sst-Cre/AAV2M1 ${ }^{\text {shRNA }}$ mice following application of muscarine alone or telenzepine followed by muscarine. (E) Proportion of DsRed+ interneurons that exhibited inward current and EPSCs after stimulation with muscarine $(n=6)$ or telenzepine followed by muscarine $(n=3)$.

tered scopolamine showed no significant effect on interaction time, providing further evidence that the antidepressant-like actions of scopolamine are mediated by M1-AChR on SST interneurons.

\section{Discussion}

Preclinical studies have started to unravel the molecular signaling pathways underlying the actions of scopolamine, but the initial cellular trigger by which scopolamine produces an antidepressant behavioral response has not been identified. In the present study, we determine whether the antidepressant effects of scopolamine are mediated through M1-AChRs on inhibitory interneurons or on glutamatergic pyramidal neurons in the $\mathrm{MPFC}$, thereby testing the indirect or direct hypothesis of action (refs. 10, 20, 21, and Figure 10). Toward this objective, we characterized M1-AChR expression in subtypes of neurons in the MPFC and developed molecular tools to selectively knock down M1-AChR levels in a neuron subtypeand brain region-specific manner. We tested the role of M1-AChR knockdown in 4 different neuron subtypes in the MPFC (Gad1Cre, Camk2a-Cre, Sst-Cre, PV-Cre) with an innovative molecular approach that provides increased temporal and spatial control compared with constitutive or conditional knockout approaches that are not brain region specific. 


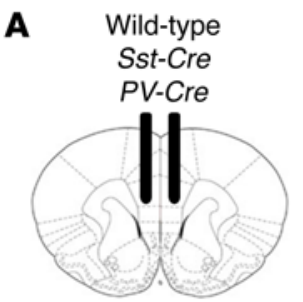

B

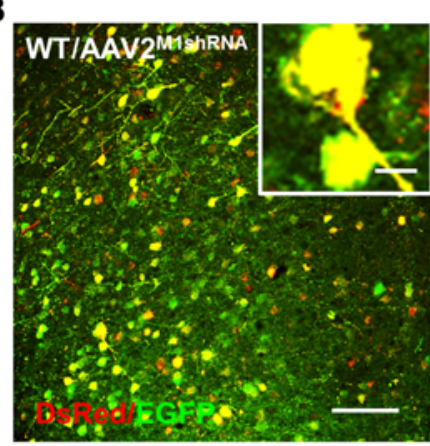

C

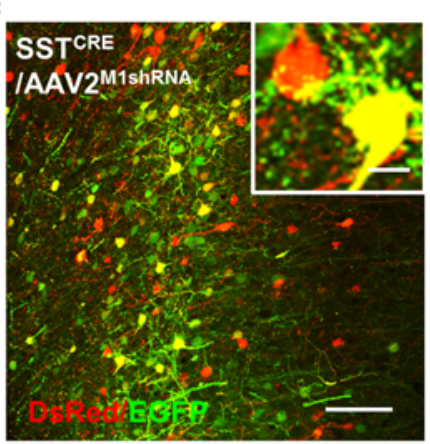

D

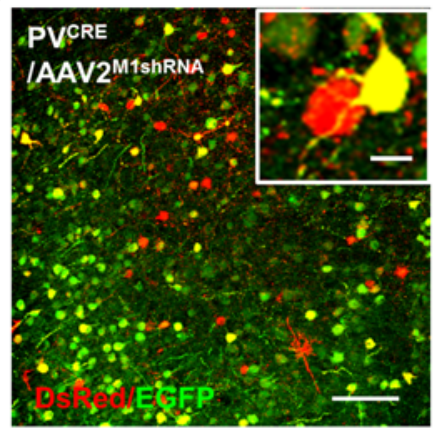

E

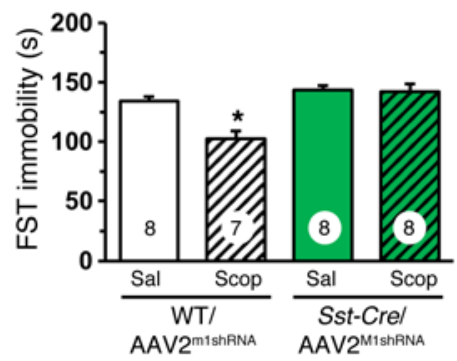

G

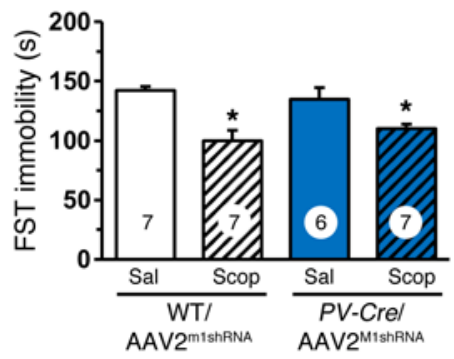

$\mathbf{F}$

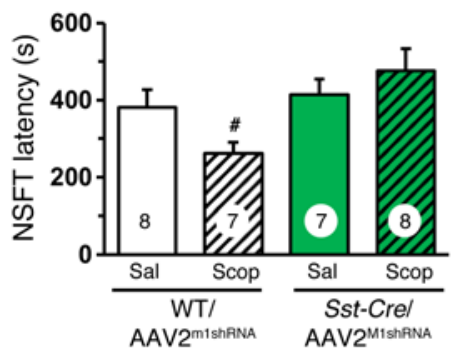

H

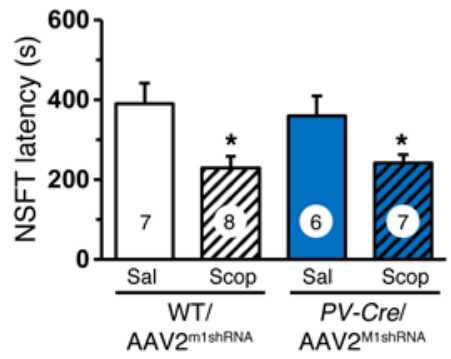

Figure 7. Infusion of AAV2 ${ }^{\mathrm{M} \text { ishRNA }}$ in mPFC of Sst-Cre mice blocked the antidepressant effects of scopolamine. WT or Sst-Cre mice received bilateral infusion of AAV2 ${ }^{\mathrm{M} 1 s h R N A}$ in the MPFC. After 3 weeks, mice were tested for baseline behavioral changes, then received saline or scopolamine, followed by additional behavioral tests. (A) Schematic showing experimental approach and time line. A subset of mice were perfused, and brains were processed for histology. (B) Representative image of AAV2 ${ }^{\text {MishRNA }}$ in prelimbic MPFC of WT mouse. Inset shows all neurons have colocalization of ECFP (green) and DsRed (red) fluorescence. Scale bars: $100 \mu \mathrm{m} ; 10 \mu \mathrm{m}$ (inset). (C and D) Representative image of AAV2 ${ }^{\mathrm{M} s h R N A}$ in prelimbic mPFC of (C) Sst-Cre or (D) PV-Cre mouse. Insets show that many neurons have colocalization of EGFP (green) and DsRed (red) fluorescence, while some have only DsRed (red) fluorescence. Scale bar: $100 \mu \mathrm{m} ; 10 \mu \mathrm{m}$ (insets). Following repeated doses of scopolamine, Sst-Cre or PV-Cre mice with corresponding WT littermates were tested in FST and NSFT. Time spent immobile in the FST (E and $\mathbf{G}$ ) and latency to feed in NSFT are shown (F and $\mathbf{H})$. Bars represent the mean \pm SEM, $n=6-8 / g r o u p$. ${ }^{*} P<0.05 ;{ }^{\#} P=0.06$, means significantly different from respective saline group. Numbers in the bars represent the total sample size for each group.

Using this brain region- and neuron-specific approach, we found that M1-AChR knockdown in MPFC of Gad1-Cre mice prevents antidepressant behavioral responses to scopolamine in the FST, NSFT, and FUST. In contrast, Camk2a-Cre mice with M1-AChR knockdown continued to show an antidepressant response to scopolamine in the FST and NSFT. M1-AChR knockdown in Gad1-Cre or Camk2a-Cre mice had no effect on baseline behaviors in the preswim test or in overall activity. These results indicate that blockade of M1-AChR on $\mathrm{GAD}^{+}$interneurons in the
mPFC is the cellular trigger for the antidepressant behavioral actions of scopolamine, supporting the indirect mechanism. These results also confirm that M1-AChR is the critical receptor subtype underlying the antidepressant actions of scopolamine $(9,23)$. It is important to note that different doses of scopolamine along with varied behavioral testing were used in the present studies compared with prior studies focused on muscarininc receptors in models of learning and memory. First, the dose $(25 \mu \mathrm{g} /$ $\mathrm{kg}$ ) is lower than in studies that report sensorimotor and cognitive 
A

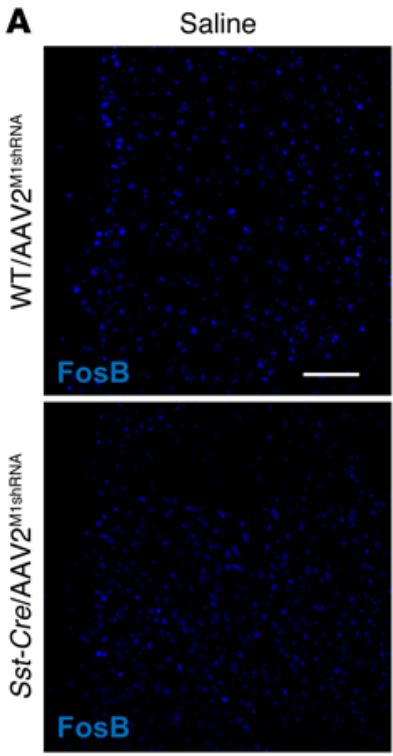

Scopolamine
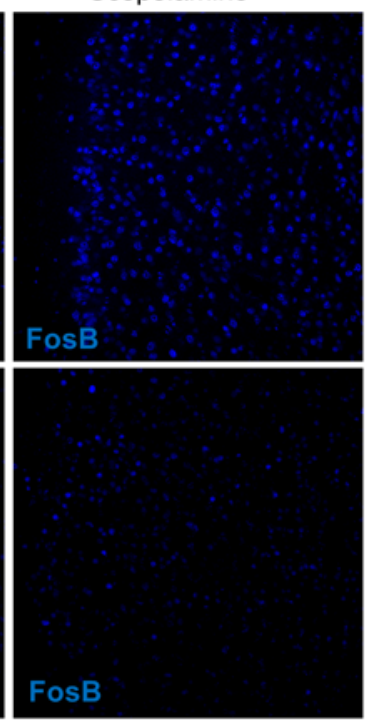

B
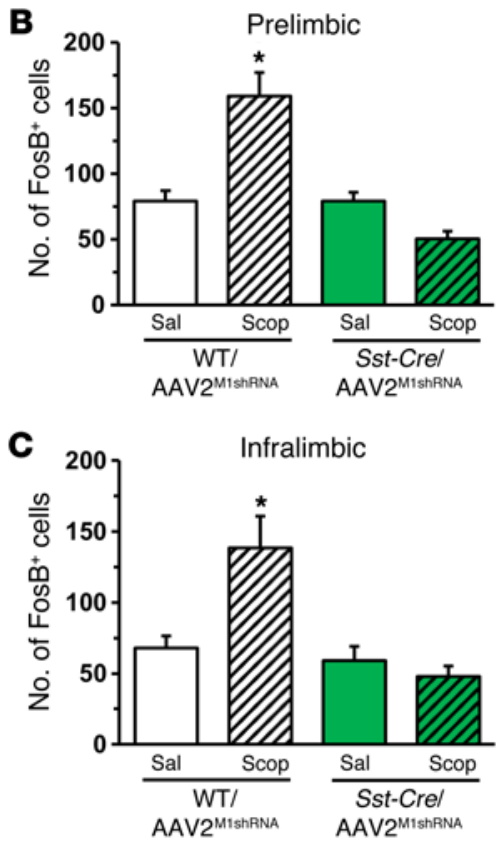

Figure 8. M1-AChR knockdown in SstCre mice decreased FosB activation following scopolamine treatment. WT or Sst-Cre mice received bilateral infusion of $A A V 2^{\mathrm{M} \text { ishRNA }}$ in the $\mathrm{mPFC}$. Following behavioral tests, mice received an acute scopolamine injection $(25 \mu \mathrm{g} / \mathrm{kg})$ and were perfused 1 hour later. Brains were collected and processed for immunohistology. (A) Representative images of FosB labeling in the prelimbic MPFC of WT/ AAV2 ${ }^{\text {MishRNA }}$ and Sst-Cre/AAV2 ${ }^{\text {MishRNA }}$ mice treated with saline or scopolamine. Scale bar: $100 \mu \mathrm{m}$. Quantification of Fos $\mathrm{B}^{+}$neurons in the (B) prelimbic or (C) infralimbic mPFC. Bars represent the mean \pm SEM, $n=4-5 /$ group. ${ }^{*} P<0.05$, means significantly different from respective saline group based on ANOVA. impairments, which typically use $100 \mu \mathrm{g} / \mathrm{kg}$ to $3 \mathrm{mg} / \mathrm{kg}$ doses of scopolamine (31-33). Second, the antidepressant-like effects were observed 24 hours after the final $25 \mu \mathrm{g} / \mathrm{kg}$ dose of scopolamine, at a time when the drug had largely been cleared.

Prior results showed that administration of scopolamine increased neuronal cFos expression in the prelimbic and infralimbic mPFC. In addition, behavioral actions of scopolamine are blocked by $\alpha$-amino-3-hydroxy-5-methyl-4isoxazolepropionic acid (AMPA) receptor antagonism or $\mathrm{GABA}_{\mathrm{A}}$ receptor agonist pretreatment, providing evidence that neuro- nal activity in the $\mathrm{MPFC}$ is required for the behavioral response to scopolamine $(9,23)$. Consistent with these data, we found that scopolamine increases FosB immunolabeling in the $\mathrm{MPFC}$ of WT mice infused with AAV2 ${ }^{\mathrm{M} 1 \text { shRNA }}$ and that this response is absent in Gad1-Cre mice with M1-AChR knockdown. These data support previous studies showing that the behavioral actions of scopolamine are dependent on neuronal activation in the MPFC and, importantly, show that neuronal activation and subsequent behavioral responses are prevented by M1-AChR knockdown on $\mathrm{GAD}^{+}$interneurons.

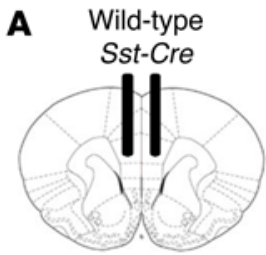

B

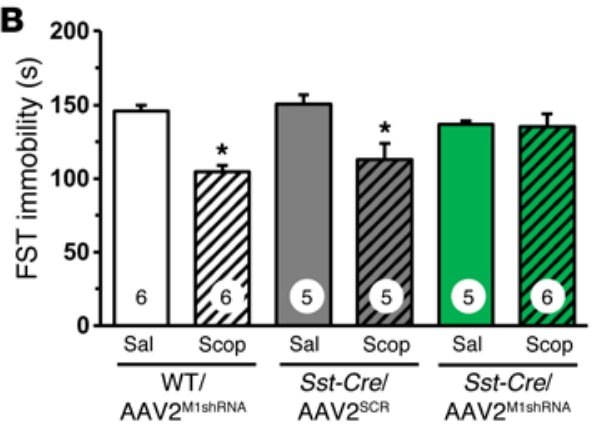

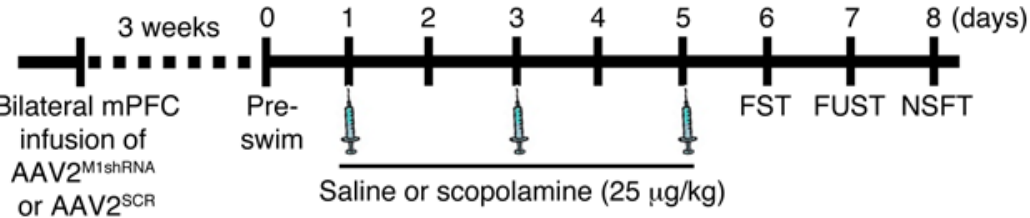

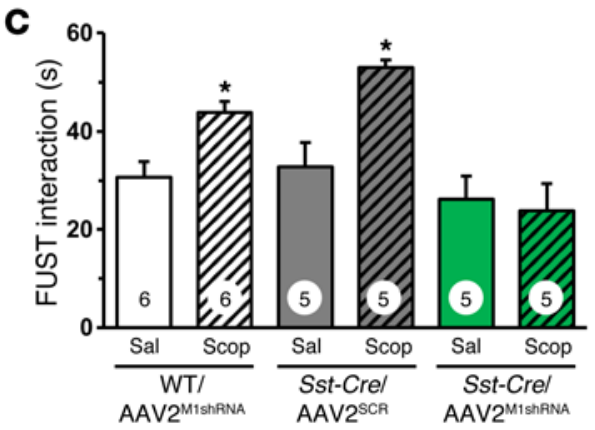

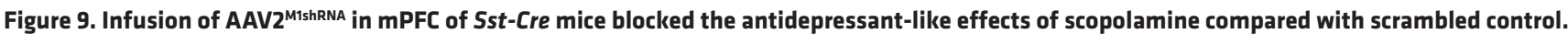
Sst-Cre mice received bilateral infusion of AAV2 ${ }^{\text {M1shRNA }}$ or AAV2 ${ }^{\text {SCR }}$ in the mPFC. After 3 weeks, mice were tested for baseline behavioral changes, then received saline or scopolamine, followed by additional behavioral tests. (A) Schematic showing experimental approach and time line. Following repeated doses of scopolamine, Sst-Cre mice were tested in FST, FUST, and NSFT. Time spent immobile in the FST (B) and interaction time in FUST (C) are shown. Bars represent the mean \pm SEM, $n=5-6$ /group. ${ }^{*} P<0.05$, means significantly different from respective saline group. Numbers in the bars represent the total sample size for each group. 


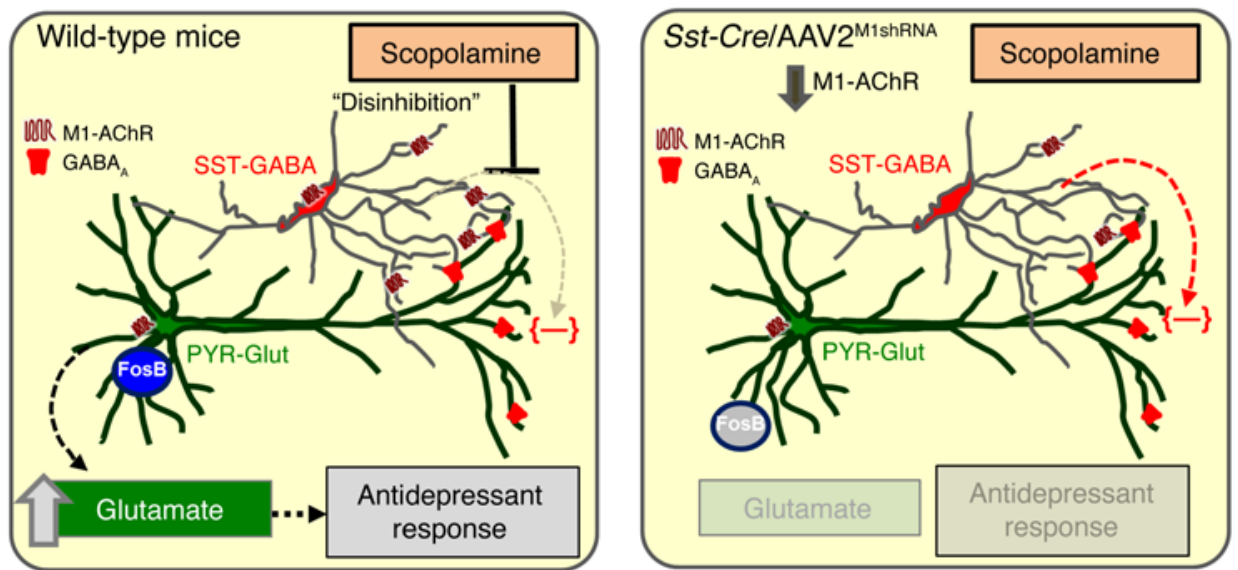

Figure 10. M1-AChR on SST interneurons mediates the rapid antidepressant response to scopolamine. In the MPFC of WT mice, M1-AChR expression on SST-GABA interneurons is antagonized by scopolamine, leading to disinhibition of pyramidal glutamate (PYR-Glut) neurons, resulting in increased glutamate release. This causes activation of surrounding target pyramidal neurons and increased FosB expression. Importantly, this burst of glutamate also stimulates postsynaptic signaling pathways that increase synaptic number and function that ultimately underlie the rapid antidepressant actions of scopolamine. In the MPFC of Sst-Cre mice, M1-AChR knockdown on SST-GABA interneurons prevents scopolamine blockade of inhibitory input on pyramidal neurons. This lack of disinhibition or maintenance of GABA inhibition of PYR-Glut neurons prevents the glutamate burst and the downstream cellular and molecular pathways underlying the synaptic and antidepressant behavioral actions of scopolamine.

GAD-expressing interneurons in the $\mathrm{MPFC}$ are diverse and display different synaptic connections, physiological properties, and molecular signaling (28). Immunohistology shows that SST interneurons have enriched expression of M1-AChR compared with PV interneurons, and electrophysiological recordings show that cholinergic stimulation of SST-tdTomato interneurons is mediated by M1-AChR. These findings correspond with previous reports showing that SST interneurons exhibit inward currents following cholinergic stimulation, while PV interneurons show minimal responses $(34,35)$. In addition, the $\mathrm{mAChR}$ antagonist pirenzepine blocked the ACh-induced inhibitory responses in the cortex, suggesting that these effects are mediated by M1-AChRs (36). SST interneurons, such as bitufted and Martinotti cells, send projections to the dendritic tufts of cortical pyramidal cells (37) and can inhibit pyramidal neuron activity directly (38). In this context, baseline cholinergic stimulation of SST interneurons through M1-AChRs leads to increased activity that would inhibit pyramidal neurons. Thus, scopolamine antagonism of M1-AChR on SST interneurons would reduce inhibitory activity, leading to disinhibition of proximal pyramidal neurons. Studies that examine cholinergic innervation of SST interneurons in the MPFC may provide additional insight into their role in the antidepressantlike effects of scopolamine.

Based on these data, we infused AAV2 ${ }^{\mathrm{M} 1 s h R N A}$ into Sst-Cre and $P V$-Cre mice to identify the GABAergic interneuron subtype that mediates the effects of scopolamine. AAV2 ${ }^{\mathrm{M} 1 \mathrm{shRNA}}$ infusion into the $\mathrm{mPFC}$ of Sst-Cre mice reduced M1-AChR immunolabeling and prevented muscarine-induced inward currents in recombined DsRed $^{+}$SST interneurons. In addition, M1-AChR knockdown in Sst-Cre mice blocked the antidepressant-like behavioral response to scopolamine in the FST and NSFT. In contrast, $P V$-Cre mice with M1-AChR knockdown displayed antidepressant-like responses to scopolamine and that this neuronal activation is dependent on M1-AChR expression in MPFC SST interneurons (Figure 10).

SST interneurons are altered following chronic stress exposure, and dysfunction of these neurons is thought to contribute to depressive symptoms $(39,40)$. Postmortem studies have reported a reduced number of calbindin ${ }^{+}$(putative SST) interneurons and decreased expression of SST interneuron markers in the PFC (41, 42), demonstrating that altered SST/GABA function is associated with depressive symptoms (43). Our prior studies indicate that scopolamine can reverse anhedonia in a chronic unpredictable stress (CUS) model of depression. In line with these studies, we provide evidence that M1-AChR on SST interneurons is necessary for increased reward-seeking behaviors in the FUST after scopolamine. Despite these findings, it will be important in future studies to determine whether M1-AChR on SST interneurons mediates antidepressant-like actions in a pathological stress model, such as CUS. Collectively, these studies indicate that mPFC interneurons have disparate expression of M1-AChR, which leads to varied responses to cholinergic stimulation. The differential response of $\mathrm{mPFC}$ interneurons to cholinergic stimulation is an important determinant of cortical network activity and can therefore influence behavioral states $(44,45)$.

In summary, the current results indicate that the rapid antidepressant responses to scopolamine are mediated by M1-AChRs on SST interneurons in the $\mathrm{MPFC}$, demonstrating that scopolamine acts via an indirect, disinhibition mechanism to regulate pyramidal neuron activity and subsequent behavioral responses. It is possible that SST interneurons also play a critical role in the rapid antidepressant-like effects of the noncompetitive NMDA receptor antagonist ketamine, as prior reports suggest that convergent cellular and molecular mechanisms underlie the behavioral responses to scopolamine and ketamine $(21,46)$. Enriched expression 
of M1-AChR on SST interneurons and disruption of SST in MDD indicate that these neurons have an important functional role in the transduction of cholinergic signaling in the cortex that influences mood and emotion as well as cognition. Additional molecular and cellular studies to examine the neurotransmitter receptor signaling profile of SST interneurons can build on these findings and provide insight into novel pharmacological targets for rapid antidepressant development.

\section{Methods}

Animals. Male transgenic mice and WT C57BL/6 littermates were obtained from in-house breeders. Gad1-Cre mice were originally obtained from Marina Picciotto (47), and Camk2a-Cre mice were obtained from Günter Schütz (German Cancer Research Center, Heidelberg, Germany) (48). PV-Cre (catalog 008069) and Sst-Cre (cata$\log$ 013044) mice were obtained from Jackson Laboratories. To obtain SST-tdTomato mice, Sst-Cre mice were bred with Ai9(RCL-tdT) mice (catalog 007909), also from Jackson Laboratories. All studies were performed with 6- to 12-week-old mice. Mice were housed in standard ventilated under a 12-hour light/12-hour dark cycle with ad libitum access to water and rodent chow.

shRNA and viral preparation. For knockdown of M1-AChR in specific neuronal cell types, an shRNA sequence was designed targeting the M1-AChR (49). M1-AChR shRNA (5'-TGCAACGCCTCTGTC ATGAATCTTCTTCAAGAGAGAAGATTCATGACAGAGGCGT TGCTTTTTTC-3') or scrambled control (5'-TATCTATAGCGACGCTCCTTGTCCATTCAAGAGATGGACAAGGAGCGTCGCTATAGATTTTTTTC-3') (Integrated DNA Technologies) was ligated into a plasmid (pM1shRNA) designed to restrict shRNA expression to cells that express Cre recombinase (50). The TATAloxP-flanked CMVEGFP cassette was subcloned from pSico (from Tyler Jacks, Massachusetts Institute of Technology, Boston, Massachusetts, USA; Addgene plasmid catalog 11578) into pAAV-DsRed (from Ralph DiLeone, Yale University School of Medicine) using HindIII and ApaI. This plasmid allows ubiquitous DsRed expression and conditional EGFP expression driven by floxed CMV cassette that prevents shRNA expression with U6 promoter disruption (Supplemental Figure 1A). This construct and scrambled control were packaged into AAV2 ${ }^{\mathrm{M} 1 \text { shRNA }}$, as previously described (19). Neuro-(N)2a cells obtained from ATCC were used to test the efficacy of pM1shRNA.

Scopolamine administration. Mice received 3 injections of vehicle or scopolamine $(25 \mu \mathrm{g} / \mathrm{kg}$, i.p.) every 48 hours, and behavioral testing was started 24 hours later (23). Following completion of behavioral testing, mice received a final injection of vehicle or scopolamine 1 hour prior to sacrifice.

Surgery and cortical infusion. Mice were anesthetized with a solution of ketamine/xylazine $(100 / 10 \mathrm{mg} / \mathrm{kg})$. Bilateral viral infusions into the $\mathrm{mPFC}(1 \mu \mathrm{l} ; 0.1 \mu \mathrm{l} /$ minute $)$ were performed with coordinates (from bregma) as follows: $+2.0 \mathrm{~mm}$ anterior-posterior, $\pm 0.2 \mathrm{~mm}$ medial-lateral, and $-2.8 \mathrm{~mm}$ dorsal-ventral (51). Incisions were closed with sutures, and an antibiotic was applied to prevent infection. Mice received i.p. injection of carprofen $(5 \mathrm{mg} / \mathrm{kg})$ immediately after surgery and daily for the next 2 days.

Anxiety-like behavior. For open-field activity, mice were placed in a Plexiglas test apparatus $(40 \times 40 \times 25 \mathrm{~cm})$ and recorded for $15 \mathrm{~min}-$ utes. Activity in the open field test was analyzed using an automated system (ANY-maze, Stoelting).
FST. FST was conducted as previously described (11). Mice were placed for 10 minutes in a clear cylinder filled with water $\left(24 \pm 1^{\circ} \mathrm{C}, 18\right.$ $\mathrm{cm}$ depth). Sessions were video recorded an $\mathrm{d}$ scored for total immobility time by a blinded experimenter. Time immobile during the 2- to 6-minute block is reported.

NSFT. NSFT was conducted as previously described (52). Mice were food deprived for 18 hours and placed in a dimly lit, novel environment $(24 \times 40 \times 14 \mathrm{~cm}$, fresh bedding) with food in the center. The latency to feed was recorded. Home cage food intake was measured as a control.

FUST. FUST is a validated test of reward-seeking behavior in rodents and was performed similarly to the way it was performed in prior reports (53). Mice were acclimated to a novel, single-housed cage for 1 hour. Following the acclimation period, mice were habituated to a water-soaked cotton-tipped applicator for 1 hour. Subsequently, a fresh water-soaked cotton-tipped applicator was inserted, and interaction time was taped for 5 minutes. The cotton-tipped applicator was removed, and mice were left undisturbed for 45 minutes. Following this time, a cotton-tipped applicator with female urine was inserted into the cage, and interaction time was taped for 5 minutes. Time spent interacting with the urine cotton-tipped applicator is presented.

Immunohistology. Brains were collected from mice after transcardiac perfusion with sterile PBS and $4 \%$ paraformaldehyde (PFA). Brains were post-fixed in 4\% PFA for 24 hours and incubated in 30\% sucrose for an additional 24 hours. Fixed brains were frozen and sectioned using a Microm HM550 cryostat. Free-floating sections were washed, then blocked for 1 hour at room temperature. Sections were washed, then incubated with the following primary antibodies (1:1,000 dilution) overnight at $4^{\circ} \mathrm{C}$ : rabbit anti-M1-AChR (Frontier Institute; mAChR-M1- RB-Af340), rat anti-Camk2a-Cre (Enzo Life Sciences; KAM-CA002-D), mouse anti-GAD67 (Sigma-Aldrich; G5419), mouse anti-PV (Millipore; MAB1572), rat anti-SST (Millipore; MAB354), and rabbit anti-FosB (Santa Cruz Biotechnology Inc.; $\mathrm{sc}-48)$. Sections were then washed and incubated with conjugated secondary antibody (1:1,000 dilution) overnight at $4^{\circ} \mathrm{C}$. Immunofluorescence was visualized using an Olympus BX61WI confocal microscope. Images were captured with Fluoview (FV1000) and a Hamamatsu high-resolution digital camera (ORCA-ER).

Quantitative immunofluorescence. For quantification of FosB, 2 to $3 \mathrm{mPFC}$ brain slices $(25 \mu \mathrm{m})$ were selected and bilateral images were obtained for each sample (all experimental groups included). Cell counts were quantified with ImageJ software (NIH). Images were transformed to 8-bit format, then threshold, particle size, and circularity were set with constant parameters used across experimental groups. Blinded counts were recorded and averaged in each cortical region, and the average across samples was used for statistical analyses $(n=4-5)$. To quantify M1-AChR colocalization with PV and SST interneurons in the MPFC, 2 to $3 \mathrm{mPFC}$ brain slices $(25 \mu \mathrm{m})$ were selected and bilateral images were obtained. The total number of PV and SST interneurons in each cortical layer was quantified along with the proportion of each subtype that colocalized with M1-AChR $(n=2-3)$. To quantify knockdown of M1-AChR through immunohistology, 2 to $3 \mathrm{mPFC}$ brain slices $(25 \mu \mathrm{m})$ were selected and immunolabeling was performed. Bilateral confocal images were collected and analyzed in ImageJ. In brief, neurons infected with $\mathrm{AAV} 2^{\mathrm{M} 1 \text { shRNA }}$ or $\mathrm{AAV} 2^{\mathrm{SCR}}$ that underwent Cre recombination were identified by DsRed expression and M1-AChR levels were measured by relative fluorescent intensity. For each sample, 6 to 8 neurons were quantified and sample averages were compiled for statistical analyses. 
RNA isolation and real-time PCR. RNA was collected from N2a cells with TRIzol reagent based on the manufacturer's protocol (54) (Life Technologies). RNA (1 $\mu \mathrm{g})$ was reverse transcribed to cDNA using the High Capacity cDNA Reverse Transcription Kit (Applied Biosystems). Primers were designed with Primer3 (Supplemental Table 1). cDNA was amplified on an ABI PRISM 7500-sequence detection system (Applied Biosystems) by real-time PCR and normalized based on reference gene expression (Gapdh). Data were analyzed with the comparative threshold cycle method.

Brain slice electrophysiology. Brain slices containing the mPFC were prepared from male and female SST-tdTomato or male Sst-Cre/ AAV2 ${ }^{\mathrm{M} 1 \text { shRNA }}$ mice (8 to 16 weeks old). Brains were placed in artificial cerebrospinal fluid (aCSF) ( $\mathrm{pH}$ 7.35-7.38) equilibrated with $95 \% \mathrm{O}_{2} / 5 \% \mathrm{CO}_{2}$. Coronal slices of $300-\mu \mathrm{m}$ thickness containing the mPFC were transferred to the fixed stage of an Olympus BX50WI scope for whole-cell recording. The chamber was continuously perfused with normal aCSF at a rate of 2 to $3 \mathrm{ml} / \mathrm{min}$ and its temperature maintained at $33 \pm 0.5^{\circ} \mathrm{C}$.

Statistics. Data were subjected to statistical analyses with GraphPad Prism 6. Significant main effects and interactions were determined using 1-way (genotype, treatment) or 2-way (genotype $\times$ treatment) ANOVA. Grouped analyses were completed without repeated measures, and multiple comparisons between group means were eval- uated with Fisher's least significant difference (LSD) test. Significant group differences were only reported if protected by significant main effects or interactions with ANOVA.

Study approval. Animal use and procedures were in accordance with NIH guidelines and approved by the Yale University Animal Care and Use Committees.

\section{Author contributions}

ESW, SRT, MRP, and RSD designed research studies. ESW, MW, and MA conducted experiments and acquired data. ESW, MW, DMG, SRT, MRP, MA, and RSD provided resources and materials. ESW, MW, MA, and RSD analyzed data and wrote the manuscript.

\section{Acknowledgments}

This research was supported by National Institute of Mental Health grants MH045481 and MH093897 (to R.S. Duman) and the state of Connecticut. We would also like to thank Riley Richmond and Xiao Li for their technical assistance.

Address correspondence to: Ronald S. Duman, 34 Park Street, New Haven, Connecticut 06520, USA. Phone: 203.974.7726; E-mail: Ronald.duman@yale.edu.
1. Kessler RC. The costs of depression. Psychiatr Clin North Am. 2012;35(1):1-14.

2. Kessler RC, Chiu WT, Demler O, Merikangas KR, Walters EE. Prevalence, severity, and comorbidity of 12-month DSM-IV disorders in the National Comorbidity Survey Replication. Arch Gen Psychiatry. 2005;62(6):617-627.

3. Murray CJ, et al. The state of US health, 19902010: burden of diseases, injuries, and risk factors. JAMA. 2013;310(6):591-608.

4. Simon GE. Social and economic burden of mood disorders. Biol Psychiatry. 2003;54(3):208-215.

5. Souery D, Papakostas GI, Trivedi MH. Treatment-resistant depression. JClin Psychiatry. 2006;67(suppl 6):16-22.

6. Drevets WC, Zarate CA Jr, Furey ML. Antidepressant effects of the muscarinic cholinergic receptor antagonist scopolamine: a review. Biol Psychiatry. 2013;73(12):1156-1163.

7. Furey ML, Khanna A, Hoffman EM, Drevets WC. Scopolamine produces larger antidepressant and antianxiety effects in women than in men. Neuropsychopharmacology. 2010;35(12):2479-2488.

8. Furey ML, Drevets WC. Antidepressant efficacy of the antimuscarinic drug scopolamine: a randomized, placebo-controlled clinical trial. Arch Gen Psychiatry. 2006;63(10):1121-1129.

9. Voleti B, et al. Scopolamine rapidly increases mammalian target of rapamycin complex 1 signaling, synaptogenesis, and antidepressant behavioral responses. Biol Psychiatry. 2013;74(10):742-749.

10. Duman RS, Aghajanian GK. Synaptic dysfunction in depression: potential therapeutic targets. Science. 2012;338(6103):68-72.

11. Li N, et al. mTOR-dependent synapse formation underlies the rapid antidepressant effects of NMDA antagonists. Science. 2010;329(5994):959-964.

12. Rajkowska G. Postmortem studies in mood disorders indicate altered numbers of neurons and glial cells. Biol Psychiatry. 2000;48(8):766-777.

13. Rajkowska G, et al. Morphometric evidence for neuronal and glial prefrontal cell pathology in major depression. Biol Psychiatry. 1999;45(9):1085-1098.

14. Drevets WC. Functional anatomical abnormalities in limbic and prefrontal cortical structures in major depression. Prog Brain Res. 2000;126:413-431.

15. Radley JJ, et al. Repeated stress alters dendritic spine morphology in the rat medial prefrontal cortex. J Comp Neurol. 2008;507(1):1141-1150.

16. Radley JJ, et al. Repeated stress induces dendritic spine loss in the rat medial prefrontal cortex. Cereb Cortex. 2006;16(3):313-320.

17. Krishnan V, Nestler EJ. The molecular neurobiology of depression. Nature. 2008;455(7215):894-902.

18. Kang HJ, et al. Decreased expression of synapserelated genes and loss of synapses in major depressive disorder. Nat Med. 2012;18(9):1413-1417.

19. Ota KT, et al. REDD1 is essential for stressinduced synaptic loss and depressive behavior. Nat Med. 2014;20(5):531-535.

20. Miller OH, Moran JT, Hall BJ. Two cellular hypotheses explaining the initiation of ketamine's antidepressant actions: Direct inhibition and disinhibition. Neuropharmacology. 2016;100:17-26.

21. Wohleb ES, Gerhard D, Thomas A, Duman RS. Molecular cellular mechanisms of rapid-acting antidepressants ketamine scopolamine. Curr Neuropharmacol. 2016;21(3):454-464.

22. Witkin JM, et al. M1 and $\mathrm{m} 2$ muscarinic receptor subtypes regulate antidepressant-like effects of the rapidly acting antidepressant scopolamine. J Pharmacol Exp Ther. 2014;351(2):448-456.

23. Navarria A, et al. Rapid antidepressant actions of scopolamine: role of medial prefrontal cortex and M1-subtype muscarinic acetylcholine receptors. Neurobiol Dis. 2015;82:254-261.

24. Disney AA, Reynolds JH. Expression of m1-type muscarinic acetylcholine receptors by parvalbumin-immunoreactive neurons in the primary visual cortex: a comparative study of rat, guinea pig, ferret, macaque, and human. J Comp Neurol. 2014;522(5):986-1003.

25. Yamasaki M, Matsui M, Watanabe M. Preferential localization of muscarinic M1 receptor on dendritic shaft and spine of cortical pyramidal cells and its anatomical evidence for volume transmission. J Neurosci. 2010;30(12):4408-4418.

26. Amar M, Lucas-Meunier E, Baux G, Fossier P. Blockade of different muscarinic receptor subtypes changes the equilibrium between excitation and inhibition in rat visual cortex. Neuroscience. 2010;169(4):1610-1620.

27. Perrotti LI, et al. Induction of deltaFosB in reward-related brain structures after chronic stress. J Neurosci. 2004;24(47):10594-10602.

28. Markram H, Toledo-Rodriguez M, Wang Y, Gupta A, Silberberg G, Wu C. Interneurons of the neocortical inhibitory system. Nat Rev Neurosci. 2004;5(10):793-807.

29. Gupta A, Wang Y, Markram H. Organizing principles for a diversity of GABAergic interneurons and synapses in the neocortex. Science. 2000;287(5451):273-278.

30. Malkesman $\mathrm{O}$, et al. The female urine sniffing test: a novel approach for assessing rewardseeking behavior in rodents. Biol Psychiatry. 2010;67(9):864-871.

31. Lieben CK, Blokland A, Sik A, Sung E, van Nieuwenhuizen P, Schreiber R. The selective 5-HT6 receptor antagonist Ro4368554 restores memory performance in cholinergic and serotonergic models of memory deficiency in the rat. Neuropsychopharmacology. 2005;30(12):2169-2179.

32. Falsafi SK, Deli A, Höger H, Pollak A, Lubec G. Scopolamine administration modulates muscarinic, nicotinic and NMDA receptor systems. PLoS One. 2012;7(2):e32082. 
33. Singh P, Konar A, Kumar A, Srivas S, Thakur MK. Hippocampal chromatin-modifying enzymes are pivotal for scopolamine-induced synaptic plasticity gene expression changes and memory impairment. J Neurochem. 2015;134(4):642-651.

34. Kawaguchi Y. Selective cholinergic modulation of cortical GABAergic cell subtypes. J Neurophysiol. 1997;78(3):1743-1747.

35. Gulledge AT, Park SB, Kawaguchi Y, Stuart GJ. Heterogeneity of phasic cholinergic signaling in neocortical neurons. J Neurophysiol. 2007;97(3):2215-2229.

36. McCormick DA, Prince DA. Two types of muscarinic response to acetylcholine in mammalian cortical neurons. Proc Natl Acad Sci U S A. 1985;82(18):6344-6348.

37. Neske GT, Patrick SL, Connors BW. Contributions of diverse excitatory and inhibitory neurons to recurrent network activity in cerebral cortex. JNeurosci. 2015;35(3):1089-1105.

38. Silberberg G, Markram H. Disynaptic inhibition between neocortical pyramidal cells mediated by Martinotti cells. Neuron. 2007;53(5):735-746.

39. Lin LC, Sibille E. Somatostatin, neuronal vulnerability and behavioral emotionality. Mol Psychiatry. 2015;20(3):377-387.

40. Soumier A, Sibille E. Opposing effects of acute versus chronic blockade of frontal cortex somatostatin-positive inhibitory neurons on behavioral emotionality in mice. Neuropsycho- pharmacology. 2014;39(9):2252-2262.

41. Sibille E, Morris HM, Kota RS, Lewis DA. GABArelated transcripts in the dorsolateral prefrontal cortex in mood disorders. Int J Neuropsychopharmacol. 2011;14(6):721-734.

42. Karolewicz B, Maciag D, O'Dwyer G, Stockmeier CA, Feyissa AM, Rajkowska G. Reduced level of glutamic acid decarboxylase-67 kDa in the prefrontal cortex in major depression. Int J Neuropsychopharmacol. 2010;13(4):411-420.

43. Northoff G, Sibille E. Why are cortical GABA neurons relevant to internal focus in depression? A cross-level model linking cellular, biochemical and neural network findings. Mol Psychiatry. 2014;19(9):966-977.

44. Picciotto MR, Higley MJ, Mineur YS. Acetylcholine as a neuromodulator: cholinergic signaling shapes nervous system function and behavior. Neuron. 2012;76(1):116-129.

45. Lawrence JJ. Cholinergic control of GABA release: emerging parallels between neocortex and hippocampus. Trends Neurosci. 2008;31(7):317-327.

46. Monteggia LM, Kavalali ET. Scopolamine and ketamine: evidence of convergence?. Biol Psychiatry. 2013;74(10):712-713.

47. Taylor SR, Badurek S, Dileone RJ, Nashmi R, Minichiello L, Picciotto MR. GABAergic and glutamatergic efferents of the mouse ventral tegmental area. JComp Neurol. 2014;522(14):3308-3334.
48. Casanova E, et al. A CamKII $\alpha$ iCre BAC allows brain-specific gene inactivation. Genesis. 2001;31(1):37-42.

49. Reynolds A, Leake D, Boese Q, Scaringe S, Marshall WS, Khvorova A. Rational siRNA design for RNA interference. Nat Biotechnol. 2004;22(3):326-330.

50. Ventura A, et al. Cre-lox-regulated conditional RNA interference from transgenes. Proc Natl Acad Sci U S A. 2004;101(28):10380-10385.

51. Paxinos G, Franklin KBJ. The Mouse Brain In Stereotaxic Coordinates. 2nd ed. San Diego, California, USA: Academic Press; 2001.

52. Elsayed M, Banasr M, Duric V, Fournier NM, Licznerski P, Duman RS. Antidepressant effects of fibroblast growth factor-2 in behavioral and cellular models of depression. Biol Psychiatry. 2012;72(4):258-265.

53. Dutheil S, Ota KT, Wohleb ES, Rasmussen K, Duman RS. High-fat diet induced anxiety and anhedonia: impact on brain homeostasis and inflammation [published online ahead of print December 14, 2015]. Neuropsychopharmacology. doi:10.1038/npp.2015.357.

54. Wohleb ES, Patterson JM, Sharma V, Quan N, Godbout JP, Sheridan JF. Knockdown of interleukin-1 receptor type-1 on endothelial cells attenuated stress-induced neuroinflammation and prevented anxiety-like behavior. J Neurosci. 2014;34(7):2583-2591. 